\title{
Papel do farmacêutico no combate ao uso indiscriminado de benzodiazepínicos: uma
}

\section{revisão de literatura}

\author{
Role of the pharmacist in fighting the indiscriminate use of benzodiazepines: a literature review \\ El papel del farmacéutico en la lucha contra el uso indiscriminado de benzodiazepinas: revisión de \\ la literatura
}

Recebido: 05/11/2021 | revisado: 15/11/2021 | aceito: 18/11/2021 | publicado: 23/11/2021

\author{
Adriano Encarnação Lima \\ ORCID: https://orcid.org/0000-0002-5601-7608 \\ Centro Universitário do Norte, Brasil \\ E-mail: adrianoencarnacaolima@gmail.com \\ Lorena Costa de Moura \\ ORCID: https://orcid.org/0000-0003-1165-7561 \\ Centro Universitário do Norte, Brasil \\ E-mail: lorenacostamoura@gmail.com \\ Yara Jéssica Bizerril Gomides \\ ORCID: https://orcid.org/0000-0003-4004-1248 \\ Centro Universitário do Norte, Brasil \\ E-mail: bizerrilgomides@gmail.com \\ Jhemerson Fernandes Paes \\ ORCID: https://orcid.org/0000-0001-5639-7041 \\ Universidade Estadual do Amazonas, Brasil \\ E-mail: jhemersonpaes@gmail.com \\ Rodrigo Queiroz de Lima \\ ORCID: https://orcid.org/0000-0002-9535-6557 \\ Centro Universitário do Norte, Brasil \\ E-mail: rodrigoq121@gmail.com
}

\begin{abstract}
Resumo
Os benzodiazepínicos são medicamentos que atuam como depressores do Sistema Nervoso Central, possuem ação anticonvulsivante, relaxante muscular, sedativa e hipnótica. Há anos estes fármacos são receitados de forma massiva, mas sem os cuidados posológicos indicados, o que leva ao uso indiscriminado do mesmo, resultando em exposição a riscos toxicológicos. Dessa maneira, o presente artigo discute, por meio de uma revisão bibliográfica integrativa, o uso indiscriminado de benzodiazepínicos e a contribuição do farmacêutico para um uso racional. O estudo foi do tipo descritivo-observacional, possuindo como metodologia a revisão de literatura integrativa. Foram selecionadas como base de dados a Biblioteca Virtual de Saúde, Biblioteca Eletrônica Científica Online e Google Acadêmico. 18 artigos foram relevantes para a pesquisa, onde percebeu-se que a administração destes medicamentos é feita majoritariamente por idosos e mulheres, relativo a questões sociais e que grande parte dos casos de uso abusivo acontece pela falta de informações de profissionais médicos e farmacêuticos. Nesse sentido,a influência do farmacêutico no combate ao uso abusivo correlacionado ao código de ética da profissão, atuará na análise dos casos de uso indiscriminado, podendo indicar a suspensão da medicação, bem como a sensibilização da comunidade e sugestões de alternativas não farmacológicas.
\end{abstract}

Palavras-chave: Ansiolíticos; Uso indevido de medicamentos; Automedicação; Relações profissional-paciente.

\begin{abstract}
Benzodiazepines are drugs that act as depressants of the Central Nervous System, have anticonvulsant, muscle relaxant, sedative and hypnotic action. These drugs have been massively prescribed for years, but without the recommended dosage care, which leads to their indiscriminate use, resulting in exposure to toxicological risks. Thus, this article discusses, through an integrative literature review, the indiscriminate use of benzodiazepines and the pharmacist's contribution to a rational use. The study was descriptive-observational, having as methodology the integrative literature review. The Virtual Health Library, Online Scientific Electronic Library and Academic Google were selected as database. 18 articles were relevant to the research, where it was noticed that the administration of these drugs is done mainly by the elderly and women, related to social issues and that most cases of abusive use occur due to lack of information from medical professionals and pharmacists. In this sense, the influence of the pharmacist in combating abusive use, correlated with the profession's code of ethics, will act in the analysis of cases of indiscriminate use, and may indicate the suspension of medication, as well as raising awareness in the community and suggesting non-pharmacological alternatives.
\end{abstract}

Keywords: Anti-anxiety agents; Drug misuse; Self medication; Professional-patient relations. 


\begin{abstract}
Resumen
Las benzodiazepinas son medicamentos que actúan como depresores del Sistema Nervioso Central, tienen acción anticonvulsivante, relajante muscular, sedante e hipnótica. Estos fármacos se prescriben masivamente desde hace años, pero sin el cuidado posológico recomendado, lo que lleva a su uso indiscriminado, resultando en exposición a riesgos toxicológicos. Así, este artículo discute, a través de una revisión integradora de la literatura, el uso indiscriminado de benzodiazepinas y la contribución del farmacéutico a un uso racional. El estudio fue descriptivoobservacional, teniendo como metodología la revisión integradora de la literatura. Se seleccionaron como base de datos la Biblioteca Virtual en Salud, la Biblioteca Electrónica Científica en Línea y el Google Académico. 18 artículos fueron relevantes para la investigación, donde se notó que la administración de estos medicamentos la realizan principalmente ancianos y mujeres, relacionados con temas sociales y que la mayoría de los casos de uso abusivo ocurren por falta de información por parte de profesionales médicos y farmacéuticos. En este sentido, la influencia del farmacéutico en el combate al uso abusivo, correlacionada con el código deontológico de la profesión, actuará en el análisis de casos de uso indiscriminado, y puede indicar la suspensión de la medicación, así como la sensibilización de la comunidad y sugiriendo alternativas no farmacológicas.
\end{abstract}

Palabras clave: Ansiolíticos; Abuso de medicamentos; Automedicación; Relaciones profesional-paciente.

\title{
1. Introdução
}

Os transtornos de ansiedade consistem em um grupo de desordens mentais caracterizadas por sentimentos de ansiedade e medo. Em 2015, a proporção da população acometida por estes transtornos foi estimada em 3,6\% em todo o mundo, em sua grande maioria mulheres (WHO, 2017). Um dos principais traços é o desencadeamento de distúrbios do sono, onde o indivíduo possui dificuldade em dormir, sendo necessário, em alguns casos, a administração de drogas com efeitos sono-induzíveis, tais como os benzodiazepínicos (Fiorelli \& Assini, 2017).

Descobertos em 1960, os benzodiazepínicos consistem em uma classe de medicamentos ansiolíticos que atuam como depressores do Sistema Nervoso Central. Agindo como agonista do principal neurotransmissor inibitório, o ácido gama-aminobutírico (GABA), eles promovem interações alostéricas com o receptor GABA tipo A (GABA-A) que culmina em uma hiperpolarização da membrana e diminuição da excitabilidade da célula (Rang et al., 2015). Como resultado, são estabelecidos os efeitos anticonvulsivante, relaxante muscular, sedativo e hipnótico desencadeados pelo medicamento (Zorzanelli et al., 2019; Votaw et al., 2020).

Nos últimos anos, esses agentes farmacológicos foram receitados de forma massiva, mas sem os esclarecimentos necessários acerca dos cuidados posológicos. Este aspecto aliado ao fácil acesso ao medicamento alocou-o na lista de fármacos administrados indiscriminadamente. O uso indiscriminado está dentro das características da automedicação e é caracterizado pelo uso sem conhecimento acerca da posologia medicamentosa, o que desencadeia o uso irracional da substância e, consequentemente, coloca o usuário em uma situação de risco a saúde, incluindo intoxicação, alteração do estado mental e dependência (Nunes \& Bastos, 2016).

Mesmo com essa dinâmica preocupante, o uso de benzodiazepínicos no Brasil aumentou de forma exponencial ao longo dos anos, tendo como principais fatores a falta de conhecimento sobre os riscos que envolvem o uso dessa classe de medicamentos, prescrições erradas e dispensação inadequada por parte do farmacêutico (Costa Filho \& Silva, 2018).

O farmacêutico ganha bastante destaque neste processo, possuindo a responsabilidade de orientar e, quando necessário, rejeitar receitas inadequadas, visando a qualidade de vida do indivíduo, como é estabelecido pelo Código de Ética da Profissão Farmacêutica (Brasil, 2021). Dentro do âmbito de controle desses agentes psicotrópicos, a ação do profissional farmacêutico deve ser ainda melhor elucidada, de forma a ultrapassar a barreira da dispensação e se tornar um agente disseminador da informação e otimizador do processo propedêutico.

Diante desta premissa, o presente artigo discute, por meio de uma revisão bibliográfica integrativa, o uso indiscriminado de benzodiazepínicos e como ocorre a contribuição do farmacêutico para um uso racional destes medicamentos, abordando pontos históricos, grupos mais susceptíveis ao uso abusivo e de que forma este profissional deve atuar dentro de um contexto tão complexo e que merece a atenção por parte dos serviços de saúde e da comunidade científica. 


\section{Metodologia}

O estudo foi do tipo descritivo-observacional, possuindo como metodologia de pesquisa e execução a revisão de literatura integrativa. Para obtenção do material de pesquisa foram selecionadas três bases de dados: a Biblioteca Virtual de Saúde (BVS), Biblioteca Eletrônica Científica Online (Scientific Electronic Library Online - SCIELO) e Google Acadêmico. A pesquisa (etapa de identificação) ocorreu por meio do uso de Descritores em Ciências da Saúde (DeCS) em português (benzodiazepínicos, psicotrópicos, ansiolíticos, hipnóticos, farmacêutico, abuso e uso racional) e Medical Subject Headings (MeSH) em inglês (benzodiazepines, psychotropic, anxiolytics, hypnotics, pharmaceuticals, abuse e rational use), os quais foram buscados de forma isolada e combinada visando obter uma quantidade adequada de material bibliográfico. Também foram consultados documentos disponibilizados pela Organização Mundial de Saúde (OMS), Órgão Internacional de Controle de Entorpecentes (International Narcotics Control Board - INCB) que dissertam sobre os transtornos de ansiedade e produção dos benzodiazepínicos, respectivamente, bem como a Resolução No 711, de 30 julho de 2021 que dispõe sobre o Código de Ética Farmacêutica.

Para melhor estratificação das informações, foram incluídos (etapa de triagem) no estudo artigos escritos em inglês ou português, publicados entre 2015 e 2021, com no mínimo dois termos de busca presentes no título (aplicados nesta respectiva ordem). Trabalhos incompletos (ausência de introdução, metodologia, resultados, discussão, conclusão ou referências bibliográficas), fora da temática trabalhada, repetidos ou com metodologias não reproduzíveis foram excluídos (etapa de elegibilidade). Após aplicação dos critérios anteriormente descritos, os estudos incluídos foram armazenados no software Mendeley Reference Managerv2.12.0 (2019) para leitura e identificação dos dados pertinentes ao trabalho. Por conseguinte, trechos e informações de interesse foram transferidos para fichas no programa Microsoft Office Word (2016) onde foram correlacionados para construção dos resultados.

\section{Resultados e Discussão}

Na mineração primária de estudos foram encontrados 358 artigos, sendo 90 da SCIELO, 121 BVS e 147 do Google acadêmico. Conseguinte a isto, tais trabalhos foram examinados quanto aos critérios de inclusão e exclusão, restando ao final do processo 18 estudos: 6 advindos da SCIELO, 4 da BVS e 8 do Google Acadêmico, como elucidado na Figura 1. Tais estudos foram a base para construção analítica das informações descritas no decorrer desta sessão.

Figura 1: Sistema para obtenção de artigos utilizados no estudo.

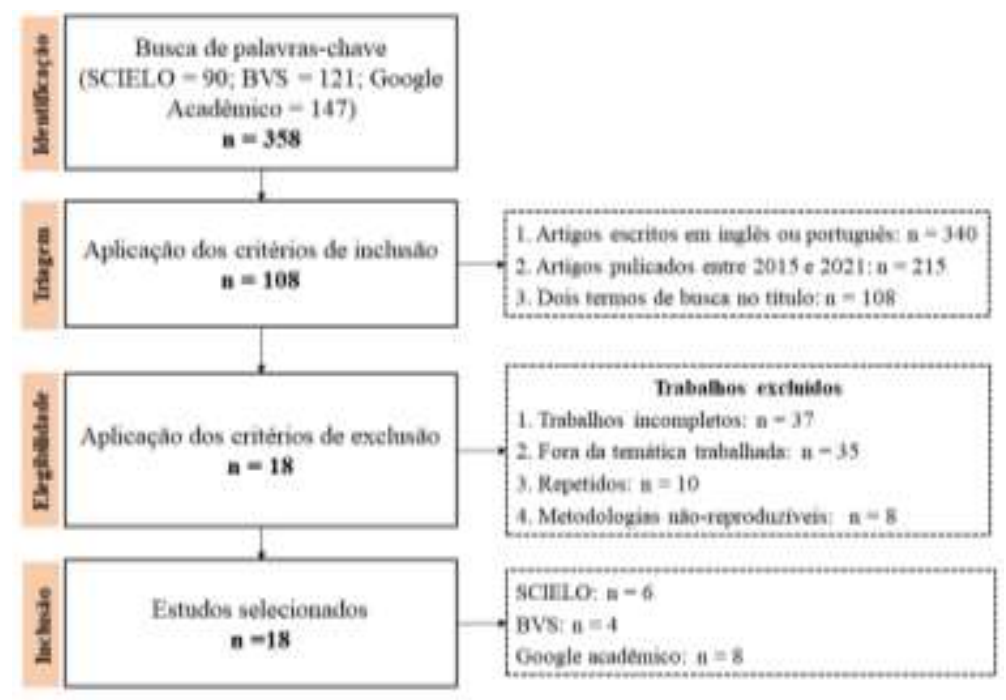

Fonte: Autores. 
A Figura 1 demonstra as etapas sistemáticas relacionadas a obtenção dos artigos. As etapas foram definidas como identificação, triagem, elegibilidade e inclusão, respectivamente. Em cada uma delas foram aplicados critérios específicos para, assim, obter resultados relevantes e úteis para o estudo desenvolvido. Ressalta-se que artigo em espanhol foram detectados durante a busca geral, os quais foram excluídos, restando 340 de artigos dos idiomas estabelecidos (inglês e português).

Os benzodiazepínicos são fármacos que contribuem para o tratamento de desordens psiquiátricas há mais de 70 anos. O primeiro fármaco desta tipologia foi descoberto em 1950, mas sua comercialização iniciou a partir da década de 1960 sob o nome de "Clordiazepóxido" (Guina \& Merrill, 2018). Por meio de sua ação hipnótica, miorrelaxante, no controle de distúrbios de ansiedade, agressividade e convulsões, o medicamento recebeu grande notoriedade entre a comunidade médica e população em geral (Azevedo, Araújo, \& Ferreira, 2016). Considerado uma promessa da terapêutica medicamentosa devido aos seus efeitos farmacológicos benéficos, sua comercialização introduziu o que a literatura especializada denominou de a "era dos benzodiazepínicos" (Nunes \& Bastos, 2016; Silva, Fernandes, \& Terra Júnior, 2018).

O início da década de 1970 veio acompanhada do vislumbre terapêutico envolvendo a ação, benefícios e efeitos pouco nocivos (identificados até aquele momento) dessas substâncias farmacológicas. Estes fatores, acompanhados do rápido crescimento da indústria farmacêutica e a busca incessante pela cura de desordens psiquiátricas, colocou esta classe de medicamentos como a prescrição médica ideal para o tratamento de distúrbios do sono e ansiedade. Todavia, a difusão deste fármaco tornou-se descontrolada entre a população americana em 1979 (chegando a 11,1\%), desencadeando os primeiros casos registrados de dependência e uso abusivo. Ao mesmo tempo, governo chileno demonstrava preocupação, descrevendo, na década de 1980, o cenário como uma questão de saúde pública (Braga et al., 2016).

A fundação americana de Administração de Alimentos e Drogas (Food and Drug Administration - FDA) restringiu, em 1975, o consumo de benzodiazepínicos visando a contenção dos casos de tolerância, dependência e uso abusivo por parte dos usuários. Em 2000, a Associação Americana de Psiquiatria (American Psychiatric Association - APA), a Organização Mundial de Saúde (OMS) e o Conselho Internacional de Controle de Entorpecentes (Internacional Narcotics Control Board INCB) reconheceram oficialmente os riscos de dependência resultantes do uso abusivo do medicamento (Guina \& Merrill, 2018; Nunes \& Bastos, 2016).

Seguindo as normas de regulamentação internacional, a Agência Nacional de Vigilância Sanitária (ANVISA) instituiu a Portaria no 344/1998, incluindo os benzodiazepínicos à lista de substâncias e medicamentos sujeitos a controle especial (ANVISA, 1998). Desta forma, o trâmite para obtenção da medicação tornou-se mais rigoroso, contudo, não extinguiu o problema relacionado ao acesso dos fármacos.

Mesmo com todos os aspectos restritivos instaurados, a diminuição no uso destes fármacos não aconteceu de imediato. Isto pode ser observado pela sua grande produção em 2000, quando mais de 300 toneladas foram fabricadas em todo o mundo. Esta elevada produção foi resultantes da alta demanda na época, demonstrando um grande problema em controlar de forma efetiva o uso destes medicamentos (INCB, 2018, 2020). Neste mesmo período, o Brasil figurou o ranking dos maiores consumidores dessas substâncias, e em de oito anos, estas substâncias farmacológicas ocuparam o $2^{\circ}$ lugar entre os medicamentos mais consumidos em território nacional (Figura 2) (Nunes \& Bastos, 2016). 
Figura 2: marcos históricos envolvendo os benzodiazepínicos.

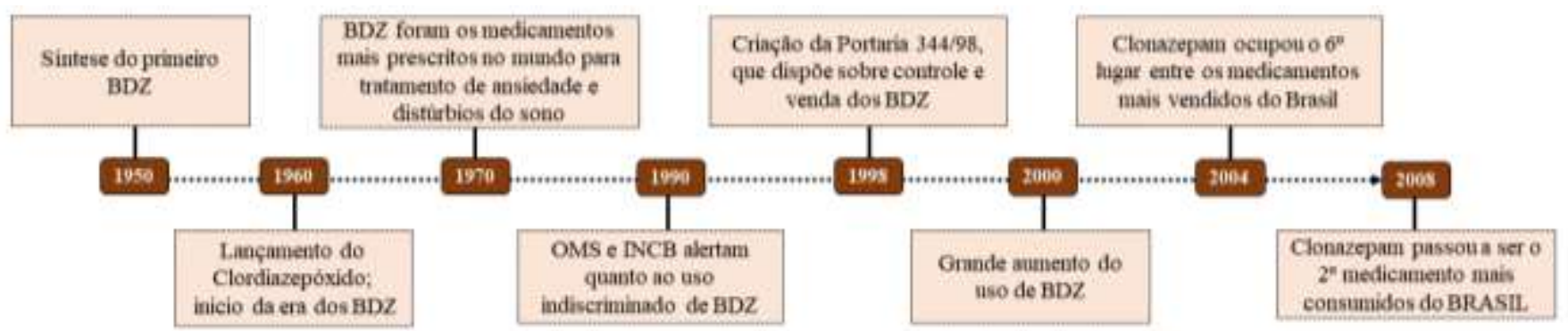

Legenda: BDZ: benzodiazepinicos: OMS: Organizaçio Mundial de Satide; INCB: Conselho Intenacional de Controle de Entorpecentes

Fonte: Autores.

A Figura 2 apresenta uma linha do tempo sobre os principais eventos mundiais envolvendo os benzodiazepínicos, onde os anos estão representados em tons mais escuros, enquanto que os eventos históricos nas caixas externas, em tons claros.

Midazolam, Diazepam, Alprazolam, e Clonazepam foram produzidos em grande escala na Itália, China, Índia, Brasil e Alemanha, respectivamente, o que correspondeu a 90\% da produção mundial de benzodiazepínicos em 2019, como observado na Figura 3 (INCB, 2020). Além de produtores, estes países também se destacaram como grandes consumidores, como dissertado por Azevedo, Araújo e Ferreira (2016) e Silva, Fernandes, e Terra Júnior (2018). A nível de Brasil, Silva, Fernandes, \& Terra Júnior (2018) cita que estes medicamentos, atualmente, estão entre os três mais consumidos, e de acordo com projeções estatísticas, este consumo pode crescer duas vezes mais a cada ano.

Figura 3: mapa dos maiores produtores de benzodiazepínicos em 2019.

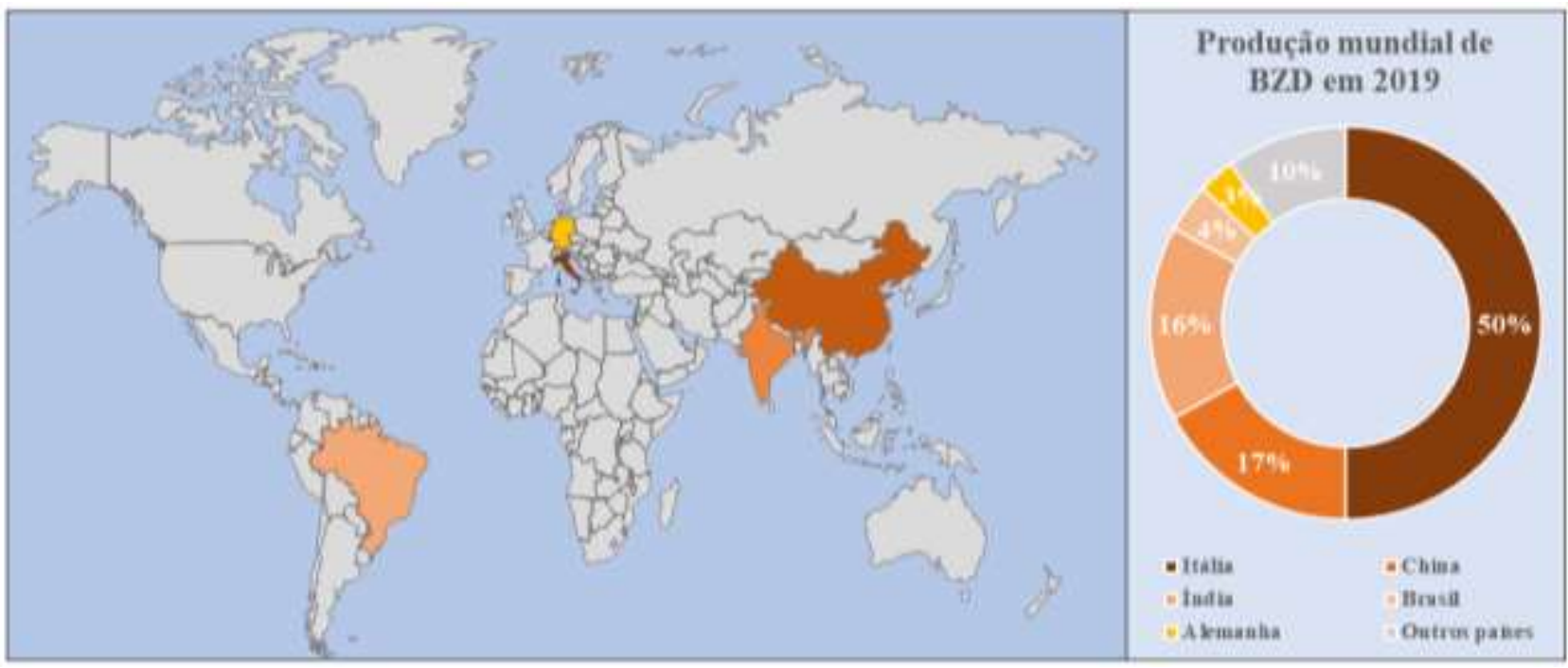

Fonte: INCB (2020) (adaptado).

A Figura 3 demonstra os principais países produtores de benzodiazepínicos em 2019. Em escala de degradê na cor laranja, variando de tons mais claro aos mais escuro, estão os países que mais produziram esses medicamentos.

\section{Uso indiscriminado e população mais acometida}

Assim como qualquer agente farmacológico, os benzodiazepínicos só devem ser administrados após prescrição médica, vendidos e dispensados com a retenção da receita (B1 e B2) como disposto na Portaria n ${ }^{\circ}$ 344/1998. Porém, esta não é a realidade encontrada ao longo dos anos nos serviços de saúde como demonstra a literatura. 
Atualmente, essa classe de medicamentos é utilizada no tratamento de distúrbios do sono, ansiedade e epilepsia (Naloto et al., 2016; Fiorelli \& Assini, 2017). Elas possuem um período de uso curto (2-4 semanas), causando indução do sono e exercendo função relaxante muscular, como anteriormente reportado. Caso a administração do fármaco persista, o usuário tende a entrar em um quadro de dependência onde mesmo ao final do tratamento a administração do medicamento se mantém contínua objetivando alcançar as sensações fornecidas pela medicação (Braga et al., 2016; Curado et al., 2021).

Essa dependência está diretamente ligada a fatores não farmacológicos e farmacológicos. Os fatores não farmacológicos estão relacionados à prescrição médica incorreta, semiologia inadequada durante a consulta, falta de instrução posológicas aos pacientes e automedicação (Azevedo, Araújo e Ferreira, 2016). Fegadolli, Varela e de Araújo Carlini (2019) ressaltam que os principais casos de prescrição incorreta acontecem na atenção básica à saúde, onde a maioria dos médicos solicitantes são clínicos gerais que, em muitos casos, não possuem conhecimento aprofundado acerca dos psicotrópicos, análise essa corroborada por Chaves e Toledo (2018), Schallemberger e Colet (2016) e Maust, Lin e Blow (2019) em seus respectivos estudos.

Os fatores farmacológicos (farmacocinética e farmacodinâmica) referem-se ao medicamento propriamente dito: tempo de meia-vida plasmática curto, lipossolubilidade, dosagem, uso prolongado e parada brusca da utilização (Kowalski, Schneider, \& Alves, 2020; Silva, Fernandes, \& Terra Júnior, 2018). Schallemberger e Colet (2016) confirmam tais condições e exploram mais a relação entre lipossolubilidade e meia-vida, dissertando que quando tais grandezas são inversamente proporcionais há grande probabilidade de se estabelecer um quadro de dependência, ou seja, quanto maior a lipossolubilidade e menor a meia-vida, maior é o a probabilidade de o fármaco causar dependência, como acontece nos benzodiazepínicos.

Uma vez dependentes, os pacientes são capazes de diversas situações para obter o medicamento. Dentre essas situações estão a falsificação de receitas, omissões sobre sintomas no momento da consulta e obtenção do medicamento com algum conhecido que trabalha em hospitais ou drogarias (Azevedo, Araújo \& Ferreira, 2016)

Contudo, o consumo destes medicamentos aumentou de maneira exponencial com o passar dos anos, como demonstrado anteriormente, e parte da literatura se debruça em esclarecer e/ou entender de que forma aconteceu este processo. Moura et al. (2017) citam o aumento dos diagnósticos de transtornos psicológicos na população como um dos responsáveis pela automedicação ao longo dos anos. Fagundes e Gonçalves (2016) complementam, citando também as grandes transformações sociais enfrentadas ao longo dos anos e problemas econômicos, apresentando questões de gênero (mulheres na busca do tratamento da ansiedade) e idade (idosos buscando alternativas farmacológicas para indução do sono), sendo estas duas últimas ressaltadas por Naloto et al. (2016), Nunes e Bastos (2016), Fiorelli e Assini (2017), Campanha e colaboradores (2020), Sousa, Mendonça, Anna e Almeida (2020), Alvim, Cruz, Aquino e Leite (2021) e Curado et al. (2021).

Azevedo, Araújo e Ferreira (2016) abordam melhor a questão social e os benzodiazepínicos, complementando o conceito idealizado por Moura et al. (2017) acerca dos diagnósticos psiquiátricos. Segundo os autores, a relação entre vulnerabilidade social e a oportunidade de se manterem sadios, a estratificação social, cenários de mortes violentas, tentativas de suicídio, falta de tratamento psicológico especializado e desemprego são fatores que convergem para o estabelecimento de uma situação instável, desencadeando problemas psiquiátricos transitórios ou permanentes.

Vale a pena resgatar as indagações de Paprocki propostas em 1990 que mesmo após 31 anos ainda são consideradas válidas para essa análise. O autor, também citado por Kowalski et al. (2020), disserta que este aumento é resultante da transição social turbulenta e não linear entre as diferentes épocas da evolução humana, levando a diminuição da resistência contra situações estressantes. Tal fator justifica a necessidade em esquecer as intempéries cotidianas a partir da utilização de alternativas farmacológicas visando refúgio e sensação de bem-estar. É uma análise multifatorial, complexa, mas indispensável para compreensão do problema, exigindo uma abordagem multidisciplinar composta por profissionais médicos, assistentes sociais e farmacêuticos. 


\section{Atuação do farmacêutico no combate ao uso indiscriminado}

Outro agente de grande importância neste contexto é o farmacêutico, profissional que intermedeia o processo entre o paciente e a medicação. O farmacêutico é capacitado para orientar os pacientes acerca da posologia dos medicamentos, diminuindo os casos de intoxicação e elevando a expectativa de vida dos mesmos. Ele é visto pelos usuários de medicamentos como uma referência, passando confiança para esclarecer suas dúvidas e garantir o uso adequado dos medicamentos (Moreira \& Borja, 2018).

Os estudos realizados por Fagundes e Gonçalves (2016), Kowalski et al. (2020), Camelo, Dinelly e Oliveira (2016), Nunes e Bastos (2016) são unânimes em confirmar a importância deste profissional na diminuição dos casos de automedicação de benzodiazepínicos. Fazendo uso da literatura científica e cumprindo os aspectos dispostos no Capítulo 3, Artigo 14, parágrafo 3 do Código de Ética da Profissão Farmacêutica, o profissional deve fornecer as orientações necessárias ao usuário, objetivando a garantia, a segurança e a efetividade terapêutica, observando o uso racional dos medicamentos (Brasil, 2021). Além disso, o mesmo também está amparado pela legislação quanto a não dispensação do medicamento em casos em que a prescrição não está adequada (situação comum em casos de benzodiazepínicos).

E a importância trespassa o ambiente hospitalar. Como profissional da saúde, o farmacêutico tem a obrigação de, baseado na literatura científica (Capítulo 3, Artigo 15, parágrafo 10), disseminar conhecimento, esclarecer mitos e prezar pela vida humana, criando uma rede de pessoas informadas que, disseminarão informação correta e de qualidade (Brasil, 2021).

Oliveira, Lopes e Castro (2015), Chaves e Toledo (2018), Moreira e Borja (2018) e Alvim, Cruz, Aquino e Leite (2021) ressaltam que este profissional pode realizar intervenção, com indicação de tratamento não farmacológico (prática de terapia comportamental), visando diminuir o uso crônico desses psicotrópicos, melhorando a qualidade de vida do paciente e otimizando a resposta farmacológica. Ações como palestras, campanhas de conscientização e maior inserção do farmacêutico dentro da saúde mental diminuiria o quantitativo de casos de uso indevido e colocaria o profissional como agente ativo na manutenção do bem estar dos usuários de benzodiazepínicos.

\section{Conclusão}

A tendência mundial ao uso abusivo de benzodiazepínicos está atrelado ao aumento dos distúrbios psiquiátricos, em especial a ansiedade e distúrbios do sono. Observou-se que o uso inadequado destes fármacos está ligado a fatores sociais, falta de conhecimento acerca dos aspectos posológicos, erros de prescrição médica, sendo este último relacionado a avaliações erradas, prescrições precipitadas ou cedimento do profissional pela pressão exercida pelo paciente. A análise também confirma o grande consumo pela população brasileira, em especial mulheres e idosos.

Observou-se que o processo de uso abusivo desses fármacos possui três possíveis agentes ativos: o médico, o paciente e o farmacêutico. Este último, como confirmado pelos dados literários, exerce papel fundamental para evitar o uso indiscriminado de tais medicamentos, sendo este também um aspecto estabelecido pela legislação que regulamenta a profissão. desta forma, este profissional pode utilizar das suas atribuições para desenvolver estratégias que visem a sensibilização da comunidade geral acerca do uso abusivo destes medicamentos bem como sugerir alternativas não-farmacológicas para terapêutica. Assim sendo, nota-se a necessidade de mais estudos envolvendo esta temática de forma a aumentar o arcabouço teórico estabelecido na literatura e otimizar o debate sobre o tema de forma estruturada e justificada por informações cientificas de confiança.

Em uma era de generalizações causadas, sobretudo, pela disseminação de informações falsas ou distorcidas, a atuação do farmacêutico apresenta-se de maneira substancial no cumprimento das prerrogativas contidas em seu código de ética, bem como a responsabilidade na prestação de informações verídicas à sociedade. 


\section{Referências}

ANVISA, S. de V. S. do M. da S. (1998). Portaria número 344 , de 12 de maio de $1998 . \quad$ Diário Oficial, 25 . http://portal.anvisa.gov.br/documents/10181/2718376/PRT_SVS_344_1998_COMP.pdf/a3ee82d3-315c-43b1-87cf-c812ba856144

Alvim, M. M., Cruz, D. T. da, Vieira, M. de T., Bastos, R. R., \& Leite, I. C. G. (2017). Prevalence of and factors associated with benzodiazepine use in community-resident elderly persons. Revista Brasileira de Geriatria e Gerontologia, 20(4), 463-473. https://doi.org/10.1590/1981-22562017020.170042

Azevedo, Â. J. P. De, Araújo, A. A. De, \& Ferreira, M. Â. F. (2016). Consumo de ansiolíticos benzodiazepínicos: Uma correlação entre dados do SNGPC e indicadores sociodemográficos nas capitais Brasileiras. Ciencia e Saude Coletiva, 21(1), 83-90. https://doi.org/10.1590/1413-81232015211.15532014

Braga, D. C., Bortolini, S. M., Pereira, T. G., Hildebrando, R. B., \& Conte, T. A. (2016). Uso de psicotrópicos em um município do meio oeste de Santa Catarina. J. Health Sci. Inst, 108-113.

Brasil. (2021). RESOLUÇÃO No 711, DE 30 JULHO DE 2021. In Diário Oficial da União.

Camelo, A. E. M., Dinelly, C. M. N., \& Oliveira, M. A. S. (2016). Psicotrópicos: Perfil De Prescrições De Benzodiazepínicos, Antidepressivos E Anorexígenos a Partir De Uma Revisão Sistemática. Revista Eletrônica de Farmácia, 13(3), 111. https://doi.org/10.5216/ref.v13i1.35226

Campanha, A. M., Ravagnani, B., Milhorança, I. A., Bernik, M. A., Viana, M. C., Wang, Y. P., \& Andrade, L. H. (2020). Benzodiazepine use in Sao Paulo, Brazil. Clinics (Sao Paulo, Brazil), 75, e1610. https://doi.org/10.6061/clinics/2020/e1610

Chaves, L. H. T., \& Toledo, D. W. F. (2018). Avaliação Do Uso De Benzodiazepínicos Em Uma Drogaria Da Cidade De Bambuí - MG INTRODUÇÃO Os benzodiazepínicos ( BDZ's ) são medicamentos psicotrópicos, dispensados apenas sob prescrição médica e retenção da receita , indicados principalmente para trat. Revista Acadêmica Conecta FASF, 3(1), 1-14.

Costa Filho, F. C. L. da C., \& Silva, H. D. M. da. (2018). Abuso De Benzodiazepínicos E Suas Consequências: Um Estudo Sistemático. Angewandte Chemie International Edition, 6(11), 951-952., 6(1), 5-24.

Curado, D. F., de Barros, V. V., Noto, A. R., \& Opaleye, E. S. (2021). Dependence on hypnotics: a comparative study between chronic users of benzodiazepines and Z-drugs. Brazilian Journal of Psychiatry, 00(00), 1-9. https://doi.org/10.1590/1516-4446-2020-1651

Fagundes, N. M., \& Gonçalves, N. M. F. M. (2016). Estudo do Grupo de Tranquilizantes Benzodiazepínicos, no Contexto da sua Utilização Abusiva e de sua Dependência. Revista UNIANDRADE, 17(3), 156-167. https://doi.org/10.18024/1519-5694/revuniandrade.v17n3p156-167

Fegadolli, C., Varela, N. M. D., \& de Araújo Carlini, E. L. (2019). Use and abuse of benzodiazepines in primary healthcare: Professional practices in Brazil and Cuba. Cadernos de Saude Publica, 35(6). https://doi.org/10.1590/0102-311X00097718

Fiorelli, K., \& Assini, F. L. (2017). A prescrição de benzodiazepínicos no Brasil: uma análise da literatura. ABCS Health Sciences, 42(1), 40-44. https://doi.org/10.7322/abcshs.v42i1.948

Guina, J., \& Merrill, B. (2018). Benzodiazepines I: Upping the care on downers: The evidence of risks, benefits and alternatives. Journal of Clinical Medicine, 7(2). https://doi.org/10.3390/jcm7020017

INCB, Internacional Narcotics Control Board. (2018). Report 2017 Press material. Unite Nations informations service, 1100(March).

INCB,Internacional Narcotics Control Board. (2020). Psychotropic Substances Substances psychotropes Sustancias sicotrópicas. In UNITED NATIONS PUBLICATION (2019o ed), Viena.

Kowalski, L., Schneider, M. S., \& Alves, I. A. (2020). Perfil dos usuários de benzodiazepínicos que frequentam uma drogaria da região Noroeste do Rio Grande do Sul. Ciência em Movimento, 22(43), 149. https://doi.org/10.15602/1983-9480/cm.v22n43p149-160

Maust, DT, Lin, LA e Blow, FC (2019). Uso e uso indevido de benzodiazepínicos entre adultos nos Estados Unidos. Serviços psiquiátricos (Washington, DC), 70 (2), 97-106. https://doi.org/10.1176/appi.ps.201800321

Moreira, P., \& Borja, A. (2018). Benzodiazepínicos: uso e abuso em pacientes idosos. Centro de Pós-Graduação, Pesquisa e Extensão Oswaldo Cruz, 10.

Moura, D., Pinto, J., Martins, P., de Arruda Pedrosa, K., \& Carneiro, M. (2017). Uso Abusivo De Psicotrópicos Pela Demanda Da Estratégia Saúde Da Família: Revisão Integrativa Da Literatura. SANARE-Revista de Políticas Públicas, 15(2), 136-144.

Naloto, D. C. C., Lopes, F. C., Barberato-Filho, S., Lopes, L. C., Del Fiol, F. de S., \& Bergamaschi, C. de C. (2016). Prescrição de benzodiazepínicos para adultos e idosos de um ambulatório de saúde mental. Ciencia e Saude Coletiva, 21(4), 1267-1276. https://doi.org/10.1590/1413-81232015214.10292015

Nunes, B. S., \& Bastos, F. M. (2016). Efeitos Colaterais Atribuídos Ao Uso Indevido E Prolongado De Benzodiazepínicos. Saúde \& Ciência Em Ação Revista Acadêmica do Instituto de Ciências da Saúde, 3(2447 9330), 71-82.

Oliveira, J. D. L. de, Lopes, L. A. M., \& Castro, G. F. P. de. (2015). Uso Indiscriminado Dos Benzodiazepínicos: a contribuição do farmacêutico para um uso consciente. Revista TRANSFORMAR, 7(7), 214-226.

Rang, H. P., Dale, M. M., Ritter, J. M., Flower, R. J., \& Henderson, G. (2015). Rang \& Dale: Farmacologia (7o ed). Recuperado de Elsevier

Schallemberger, J. B., \& Colet, C. de F. (2016). Avaliação da dependência e da ansiedade em usuários de benzodiazepínicos em um município do interior do Rio Grande do Sul. Trends in Psychiatry and Psychotherapy, 38(2), 63-70. https://doi.org/10.1590/2237-6089-2015-0041

Silva, E. G., Fernandes, D. R., \& Terra Júnior, A. T. (2018). Uma Abordagem Ao Uso Indiscriminado De Medicamentos Benzodiazepínicos. Revista Científica FAEMA, 9(edesp), 610-614. https://doi.org/10.31072/rcf.v9iedesp.591 
Research, Society and Development, v. 10, n. 15, e304101522886, 2021

(CC BY 4.0) | ISSN 2525-3409 | DOI: http://dx.doi.org/10.33448/rsd-v10i15.22886

Sousa, G. dos S., Mendonça, R. V. de M., Anna, C. de C. S., \& Almeida, M. K. C. de. (2020). As Consequências E Os Efeitos Decorrentes Do Uso Indiscriminado E Prolongado De Benzodiazepínicos - Compostos Bdz: Uma Revisão De Literatura. Revista Amazônica de Ciências Farmacêuticas, 1(2), 5469. https://doi.org/10.17648/2675-5572.racf.v1n1.2-4

Votaw, V. R., Geyer, R., Rieselbach, M. M., \& McHugh, R. K. (2019). The epidemiology of benzodiazepine misuse: A systematic review. Drug and alcohol dependence, 200, 95-114. https://doi.org/10.1016/j.drugalcdep.2019.02.033

WHO,World Health Organization. (2017). Depression and Other Common Mental Disorders. WHO Document Production Services, 48(1), 56-60.

Zorzanelli, R. T., Giordani, F., Guaraldo, L., De Matos, G. C., De Brito Junior, A. G., De Oliveira, M. G., \& Rozenfeld, S. (2019). Consumption of the benzodiazepine clonazepam (Rivotrilß) in rio de janeiro state, brazil, 2009-2013: An ecological study. Ciencia e Saude Coletiva, 24(8), 3129-3140. https://doi.org/10.1590/1413-81232018248.23232017 